\title{
Plantaricins markedly enhance the effects of traditional antibiotics against Staphylococcus epidermidis
}

\author{
Robert Selegård ${ }^{\ddagger}, 1$, Amani Musa ${ }^{\ddagger}{ }^{1}$, Pontus Nyström ${ }^{1}$, Daniel Aili², Torbjörn Bengtsson ${ }^{1}$ \& \\ Hazem Khalaf*, 1 \\ ${ }^{1}$ Faculty of Medicine \& Health, School of Medical Sciences, Örebro University, Örebro, Sweden \\ 2Division of Molecular Physics, Department of Physics, Chemistry \& Biology (IFM), Linköping University, Linköping, Sweden \\ *Author for correspondence: hazem.khalaf@oru.se \\ ${ }^{\ddagger}$ Authors contributed equally
}

\begin{abstract}
Aim: Bacteriocins are considered as promising alternatives to antibiotics against infections. In this study, the plantaricins (PIn) A, E, F, J and K were investigated for their antimicrobial activity against Staphylococcus epidermidis. Materials \& methods: The effects on membrane integrity were studied using liposomes and viable bacteria, respectively. Results: We show that PInEF and PInJK caused rapid and significant lysis of S. epidermidis, and induced lysis of liposomes. The PInEF and PInJK displayed similar mechanisms by targeting and disrupting the bacterial cell membrane. Interestingly, Pln enhanced the effects of different antibiotics by 30 - to 500 -fold. Conclusion: This study shows that PIn in combination with low concentrations of antibiotics is efficient against $S$. epidermidis and may be developed as potential treatment of infections.
\end{abstract}

First draft submitted: 19 October 2018; Accepted for publication: 21 December 2018; Published online: 16 January 2019

Keywords: antibiotic $\bullet$ combination therapy $\bullet$ liposome $\bullet$ membrane lysis $\bullet$ plantaricin $\bullet$ Pln $\bullet$ Staphylococcus epidermidis • synergy

Staphylococcus spp., including strains that are resistant to multiple antibiotics, are one of the leading causes of community and hospital-acquired bacterial infections [1]. These bacteria can cause superficial infections and severe infections associated with chronic wounds and insertion of medical devices, including catheters and prosthetic joint implants [2]. This may subsequently increase the risk for development of life-threatening conditions, such as sepsis [3,4]. Hand hygiene and infection control strategies, including active surveillance programs, are important to prevent bacterial transmission in hospitals [5]. However, given the rise in antimicrobial resistance, the development of new strategies to combat bacterial infections are urgently needed, and bacteriocins represent a promising alternative $[6,7]$.

Several strains of Lactobacillus plantarum are probiotic strains currently used as dietary supplements and have been reported to express several plantaricins (Pln) that belongs to class IIb [8]. Bacteriocins, including plantaricins, are antimicrobial peptides that are secreted by many bacteria as part of their defense mechanism. This group of antimicrobial peptides displays low toxicity toward eukaryotic cells and is active against pathogens and bacteria that have acquired resistance to antibiotics $[6,9]$. We have recently shown that the plantaricin PLNC8 $\alpha \beta$ efficiently permeabilizes the membrane of the periodontal pathogen Porphyromonas gingivalis [10], and antagonizes the cytotoxic effect of this pathogen on host cells [11]. All $p \ln$ genes, including $p \ln E F$ and $p \ln J K$, are adjacent to each other and located within the pln locus of the L. plantarum genome. The two-peptide plantaricins, PlnEF and PlnJK, have been suggested to kill microbes through formation of pores $[8,12,13]$. These mechanisms are difficult to evade and to develop resistance against, compared with conventional antibiotics that usually target metabolic enzymes. A possible approach that may be successful against bacterial infections is combination therapy. It has been suggested that inhibition of multiple bacterial targets is an effective method and could potentially delay selection of resistance while reducing the dosage and thus possible side effects [14,15]. A recent study reported that the bacteriocin garvicin KS acted synergistically with other bacteriocins, including polymyxin B and nisin, against a broad range of Gram- 
Table 1. Amino acid sequences and molecular weight of plantaricins.

\begin{tabular}{|ll|}
\hline Plantaricin & Amino acid sequence \\
\hline E & AYSLQMGATAIKQVKKLFKKWGW \\
\hline F & FNRGGYNFGKSVRHVVDAIGSVAGIRGILKSIR \\
\hline J & VFHAYSARGVRNNYKSAVGPADWVISAVRGFIHG \\
\hline K & GAWKNFWSSLRKGFYDGEAGRAIRR \\
\hline
\end{tabular}

positive and Gram-negative bacteria [16]. Furthermore, the lantibiotic nisin has been shown to act synergistically with citric acid [17] and with traditional antibiotics, including penicillin and chloramphenicol [18], against several staphylococci strains.

It is important to find alternative antimicrobial candidates against staphylococci, which are responsible for causing the most common bacterial infections in humans. Although bacteriocins have received increased attention for applications in clinical settings, more research is needed to understand their mechanisms of action and effects on resistance development. The aim of the present study was to elucidate the antimicrobial activity of plantaricins, with and without traditional antibiotics, against Staphylococcus epidermidis.

\section{Materials \& methods}

Bacterial culture conditions

Staphylococcus epidermidis ATCC 12228 (ATCC, VA, USA) and a clinical isolate, S. epidermidis 126, that was found to have heterogeneous resistance against glycopeptide antibiotics, gentamicin and tetracycline, were grown on Luria-Bertani agar plates and incubated at $37^{\circ} \mathrm{C}$ overnight. Single colonies were inoculated into $5 \mathrm{ml}$ of Luria-Bertani broth and incubated on a shaker $\left(300\right.$ r.p.m.) at $37^{\circ} \mathrm{C}$ overnight. The bacterial concentration was determined by viable count by culturing on agar plates and was adjusted to correlate with approximately $10^{9}$ $\mathrm{CFU} / \mathrm{ml}$.

\section{Peptide synthesis}

All chemicals were bought from Sigma-Aldrich unless otherwise noted and used without further purification. The peptides, PlnA, E, F, J and K (Table 1) were synthesized using conventional Fmoc chemistry on a Quartet automated peptide synthesizer (Protein Technologies, Inc., AZ, USA) in a $100 \mu \mathrm{mol}$ scale. The C-terminal amino acid of each peptide were attached to a Wang resin (Novabiochem, $1.13 \mathrm{mmol} / \mathrm{g}$ ) using 5 equivalents (eq) of Fmoc-protected amino acid (Iris biotech gmbh), 5 eq of MSNT and 3.75 eq of Melm in dry dichloromethane (DCM). The reactions were allowed to proceed for $1 \mathrm{~h}$ in a $\mathrm{N}_{2}$ atmosphere. Resins were filtered off and washed with DCM and the loading procedures were repeated once. Peptide elongation was performed using a 4 eq of amino acid and activator (TBTU, Iris biothech gmbh) and using 8 eq of base (DIPEA). The Fmoc removal was accomplished by treatment with Piperidine (20\% in DMF, v/v). All peptides were cleaved from their solid support using a mixture of TFA, triisoproylsilane and water (95:2.5:2.5, v/v/v) for $2 \mathrm{~h}$ before being, filtered, concentrated and precipitated twice in cold diethylether. Crude peptides were purified on a C-18 reversed phase column (Kromatek HiQ-Sil C18HS) attached to a semipreparative HPLC system (Dionex) using an aqueous gradient of acetonitrile (10-46\%) containing $0.1 \%$ TFA. Mass identity of all peptides was confirmed by MALDI-ToF MS (UltraflexXtreme, Bruker Daltonics) using $\alpha$-cyano-4-hydroxycinnamic acid as matrix (Supplementary Figure 1). All peptide stock solutions were diluted with $\mathrm{ddH}_{2} \mathrm{O}$ in the same manner to minimize differences in error between samples.

\section{Liposome preparation}

Liposomes were formed according to methods that are well established in the field [19,20]. Briefly, liposomes were prepared by dry film formation, hydration and finally extrusion through a polycarbonate membrane to form monodisperse large unilamellar vesicles. The lipids 1-palmitoyl-2-oleoyl-sn-glycero-3-phospho-L-serine (POPS) and 1palmitoyl-2-oleoyl-sn-glycero-3-phosphatidylcholine (POPC) (Avanti Polar Lipids, AL, USA) were mixed at a molar ratio of 5:95 while dissolved in chloroform. A dry lipid film was formed by evaporation of the chloroform by nitrogen flow and dried overnight at reduced pressure. The film was hydrated with phosphate buffer $(10 \mathrm{mM}$, $\mathrm{pH}$ 7.4) containing self-quenching concentration of 5(6)-carboxyfluorescein (CF, $50 \mathrm{mM}$ ) for fluorescence leakage assays and phosphate buffer saline (PBS, $10 \mathrm{mM}, \mathrm{pH}$ 7.4) for circular dichroism (CD) measurements. The solu- 
tions were vortexed for $1 \mathrm{~min}$ and put on a shaker for $1 \mathrm{~h}$ before extruded 21-times through a 100-nm pore-sized polycarbonate membrane. Liposomes used in fluorescence leakage assays were purified from unencapsulated CF by gel filtration using a PD-25 column (GE Healthcare, Uppsala, Sweden) and eluted with PBS.

\section{Circular dichroism spectroscopy}

Bacteriocins are often unstructured in solution but typically adopt a more ordered secondary structure when bound to the bacterial cell membrane $(\mathrm{CM})$ as a result of membrane partitioning [21]. The secondary structure of the bacteriocins $(100 \mu \mathrm{M}, \mathrm{PBS})$ alone and in combinations with and without liposomes $(0.5 \mathrm{mg} / \mathrm{ml}, 660 \mu \mathrm{M})$ was investigated using CD spectroscopy. Spectras were recorded using a Chirascan spectropolarimeter (Applied Photophysics, UK) and a $0.1-\mathrm{mm}$ quartz cuvette at $25^{\circ} \mathrm{C}$ with a sampling interval of $0.5 \mathrm{~nm}$. All measurements were done in triplicates and averaged before converted to mean residue ellipticity and curves were smoothened using Savitzky-Golay algorithm.

\section{Carboxyfluorescein release assay}

Leakage of the liposome-encapsulated fluorophore CF due to additions of Pln peptides were recorded using a fluorescence plate reader (Safire 2, Tecan, Austria) with $\lambda_{\mathrm{ex}}=492 \mathrm{~nm}$ and $\lambda_{\mathrm{em}}=517 \mathrm{~nm}$. The CF was encapsulated at self-quenching concentration and CF release results in an increased fluorescence signal. Liposomes were diluted to $25 \mu \mathrm{M}$ (total lipid concentration) in PBS, followed by additions of Pln peptides $(0.005-100 \mu \mathrm{M})$, alone and in different combinations and incubated for $30 \mathrm{~min}$ prior to measurements. In order to estimate the maximum release from each sample, a final addition of 1\% Triton X-100 was made at the end of all measurements and the total amount of CF (100\% release) was estimated after 10-min incubation. All data were fitted using a monophasic Hill equation and the concentration of Pln peptides needed to obtain a 50\% CF release was extracted.

\section{Microscopy}

The fluorescent dye Sytox ${ }^{\circledR}$ Green binds to nucleic acids and fluoresces only after having crossed damaged membranes, was used to study the permeabilizing activity of plantaricins on S. epidermidis. The bacteria were washed and resuspended in Krebs-Ringer buffer $(120 \mathrm{mM} \mathrm{NaCl}$, 4.9 mM KCl, $1.2 \mathrm{mM} \mathrm{MgSO}, 1.7 \mathrm{mM}$ $\mathrm{KH}_{2} \mathrm{PO}_{4}$ and $\left.8.3 \mathrm{mM} \mathrm{Na}_{2} \mathrm{HPO}_{4}, \mathrm{pH} 7.3\right)$ and incubated in the presence or absence of different combinations of plantaricins in 96-well microtiter plates for 2 min. Images were captured with Olympus BX41 at $40 \times$ magnification. Fluorescence intensity of Sytox Green was quantified using the software ImageJ.

Transmission electron microscopy was used to visualize the effects of different Pln peptides on S. epidermidis. Briefly, the bacteria were pelleted and washed with Krebs-Ringer buffer followed by exposure to different plantaricins at a final concentration of $25 \mu \mathrm{M}$ for $5 \mathrm{~min}$. Samples were fixed in $2.5 \%$ glutaraldehyde in $0.1 \mathrm{M}$ phosphate buffer, $\mathrm{pH}$ 7.3. The samples were then washed in $0.1 \mathrm{M}$ phosphate buffer and postfixed in $2 \%$ osmium tetroxide in $0.1 \mathrm{M}$ phosphate buffer for $2 \mathrm{~h}$ and embedded into LX-112 (Ladd, VT, USA). Ultrathin sections ( 50-60 nm) were cut by a Leica ultracut UCT/ Leica EM UC 6 (Leica, Wien, Austria). Sections were contrasted with uranyl acetate followed by lead citrate and examined in a Hitachi HT 7700 (Tokyo, Japan). Digital images were taken by using a Veleta camera (Olympus Soft Imaging Solutions, GmbH, Münster, Germany).

\section{Detection of ATP release}

The concentration of extracellular ATP, which was used as a marker of bacterial lysis, was recorded using a luciferin/luciferase bioluminescence assay (Sigma-Aldrich, MO, USA) in bacterial suspensions $\left(2.5 \times 10^{8}\right.$ $\mathrm{CFU} / \mathrm{ml}$ ). The bacteria were exposed to different concentrations of PlnEF and PlnJK, and real-time changes in bioluminescence were recorded in a Chronolog lumi-aggregometer (Chrono-Log, PA, USA) for 10 min. The levels of ATP were calculated based on the bioluminescence signals recorded in response to known concentrations of ATP.

\section{Antimicrobial activity of plantaricins}

The broth microdilution method was used to determine MIC and minimal bactericidal concentration (MBC). Twofold serial dilutions of the peptides were used and the final concentrations ranged from 0.097 to $50 \mu \mathrm{M}$ $(\sim 0.34-177 \mu \mathrm{g} / \mathrm{ml})$. Since the activity of PlnEF and PlnJK is dependent on the complementary action of two peptides at equimolar concentrations, a final concentration of $50 \mu \mathrm{M}$ of, for example, PlnEF is achieved by mixing $50 \mu \mathrm{M}$ of PlnE with $50 \mu \mathrm{M}$ PlnF. The final concentrations of the antibiotics vancomycin and teicoplanin ranged from 0.097 to $50 \mu \mathrm{g} / \mathrm{ml}$, while gentamicin ranged from 0.0097 to $5 \mu \mathrm{g} / \mathrm{ml}$ and tetracycline from 0.016 to $8 \mu \mathrm{g} / \mathrm{ml}$. 
The antimicrobial effects of plantaricins together with antibiotics were studied by using the same concentration series of antibiotics with a constant concentration of plantaricins in all the wells $(12.5$ and $6.25 \mu \mathrm{M}$ equivalent to 44 and $22 \mu \mathrm{g} / \mathrm{ml}$, respectively). The MIC was determined visually and spectroscopically $(620 \mathrm{~nm})$ as the first concentration that completely inhibited bacterial growth. All concentrations that resulted in complete inhibition of bacterial growth were cultured $(10 \mu \mathrm{l})$ on blood-agar plates, and the lowest concentration where no growth was observed on agar represented the MBC. All experiments were repeated at least three-times.

\section{Statistical analysis}

All data were analyzed using GraphPad Prism 5.0 (GraphPad Software, CA, USA). One-way ANOVA with Bonferroni's post hoc test was used for the comparisons between the different treatments. The p-values are referred to as ${ }^{*} \mathrm{p}<0.05 ;{ }^{* *} \mathrm{p}<0.01 ;{ }^{* * *} \mathrm{p}<0.001$.

\section{Results}

\section{Effects of plantaricins on S. epidermidis \& model lipid membranes}

The antimicrobial effects of plantaricins were tested against one of the most common opportunistic pathogens found in humans, in other words, S. epidermidis. The inhibitory and bactericidal concentrations of the different plantaricins were determined by the broth microdilution method. S. epidermidis was more susceptible to PlnEF (Figure 1A) than PlnJK (Figure 1B), with a MIC/MBC value of $12.5 / 25$ and 25/50 $\mu \mathrm{M}$, respectively. However, the individual plantaricin peptides (PlnE, F, J and $\mathrm{K}$ ) were ineffective at inhibiting bacterial growth. Furthermore, the two-peptide bacteriocins PlnEF and PlnJK, at 25 and $50 \mu \mathrm{M}$, caused a significant increase in turbidity immediately after addition to a bacterial suspension, which is indicative of rapid bacterial lysis (data not shown). The PlnA $(50 \mu \mathrm{M})$ alone displayed antimicrobial activity that suppressed bacterial growth; however, the concentration was not bactericidal.

Plantaricins of $L$. plantarum are short peptides with membrane perturbing properties. By probing the secondary structure with CD spectroscopy, we found that individual Pln peptides adopts a distorted conformation except PlnJ which resides as a distinct $\alpha$-helix in PBS buffer at $\mathrm{pH} 7$ (Supplementary Figure 2). The presence of a model lipid membrane had little to no influence on individual Pln peptides except PlnA and J. However, the combination of different peptides (PlnEF, AEF, JK and AJK) resulted in more pronounced induced helicity. It has previously been shown that the lipid composition of the model membrane is critical for the formation of helical secondary structure as well as the overall structure of the model membrane, where micelles tend to induce more prominent helixes compared with liposomes [22]. We, however, used large unilamellar liposomes with a size of $100 \mathrm{~nm}$ composed of the anionic lipid 1-palmitoyl-2-oleoyl-sn-glycero-3-phospho-L-serine (POPS) and zwitterionic 1-palmitoyl-2oleoyl-sn-glycero-3-phosphatidylcholine (POPC) at a molar ratio of 5:95 as a model system in order to provide a more realistic model membrane. This membrane model system was used to investigate the permeabilizing effect of the Pln peptides by using liposomes loaded with the fluorophore CF at a self-quenching concentration. The relative CF release was recorded $30 \mathrm{~min}$ after exposure of liposomes to increasing concentrations of different Pln peptides. The CF release was significantly increased by PlnEF (Figure 1C) and PlnJK (Figure 1D), and reached $50 \% \mathrm{CF}$ release at 0.33 and $0.20 \mu \mathrm{M}$, respectively, compared with each individual peptide alone that caused a $50 \%$ $\mathrm{CF}$ release at concentration $\geq 30 \mu \mathrm{M}$. The PlnA alone was efficient and caused lysis of liposomes at concentrations equivalent to PlnEF. Interestingly, addition of PlnA to EF or JK resulted in a synergistic lytic effect of liposomes with a $50 \%$ CF release of 0.028 and $0.025 \mu \mathrm{M}$, respectively.

\section{Plantaricins efficiently permeabilize S. epidermidis \& cause extensive morphological changes}

The ability of plantaricins to permeabilize gram-positive S. epidermidis ATCC was evaluated with Sytox Green, which only migrates through damaged membranes, binds to nucleic acid and fluoresces. The PlnA alone, but not PlnE, F, J or K, was able to permeabilize the bacteria (Figure 2A \& B). The combinations PlnEF and PlnJK caused rapid permeabilization $(2 \mathrm{~min})$ in a dose-dependent manner. Interestingly, addition of PlnA to EF or JK caused formation of larger bacterial aggregates, probably due to a synergistic antimicrobial effect of the plantaricins. The antimicrobial effects of plantaricins were verified by quantifying ATP release from S. epidermidis after treatment with different concentrations of PlnJK and PlnEF (Figure 2C). Both PlnJK and PlnEF caused rapid lysis that peaked after 2 and $4 \mathrm{~min}$, respectively. The bacteria were lysed in a dose-dependent manner and PlnEF was more effective than PlnJK. As these plantaricins showed different abilities to lyse liposomes and S. epidermidis, we studied the ultrastructural changes that are associated with plantaricin-induced bacterial lysis. Transmission electron 
(A)

\begin{tabular}{lcc}
\hline Plantaricin & $\begin{array}{c}\text { MIC } \\
(\mu \mathrm{M})\end{array}$ & $\begin{array}{l}\text { MBC } \\
(\mu \mathrm{M})\end{array}$ \\
\hline \hline A & 50 & $>50$ \\
E & $>50$ & $>50$ \\
F & $>50$ & $>50$ \\
EF & 12.5 & 25 \\
AEF & 25 & 25 \\
\hline
\end{tabular}

(c)

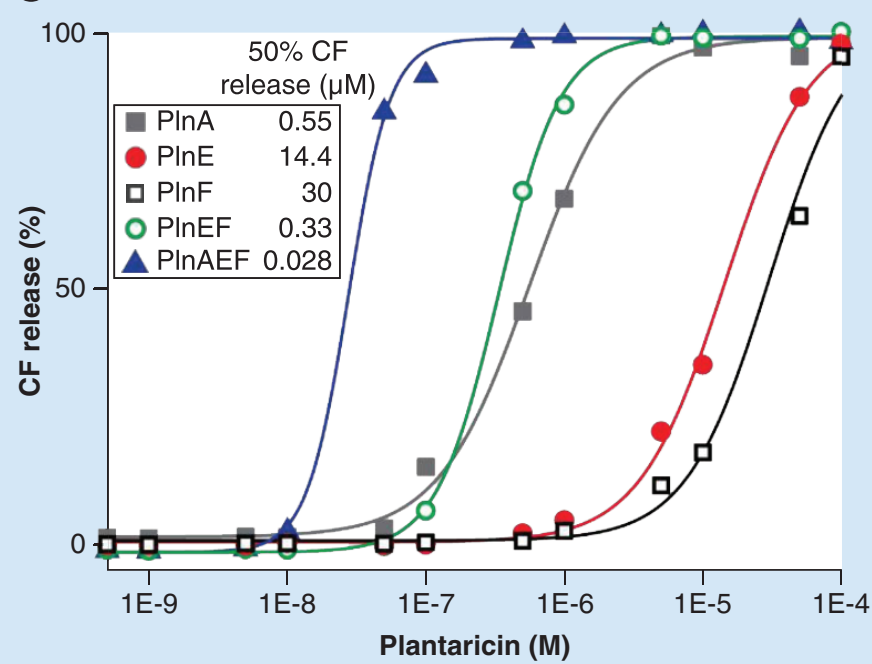

(B)

\begin{tabular}{lcc}
\hline Plantaricin & $\begin{array}{c}\text { MIC } \\
(\mu \mathrm{M})\end{array}$ & $\begin{array}{c}\text { MBC } \\
(\boldsymbol{\mu M})\end{array}$ \\
\hline \hline A & 50 & $>50$ \\
J & $>50$ & $>50$ \\
K & $>50$ & $>50$ \\
JK & 25 & 50 \\
AJK & 25 & 25 \\
\hline
\end{tabular}

(D)

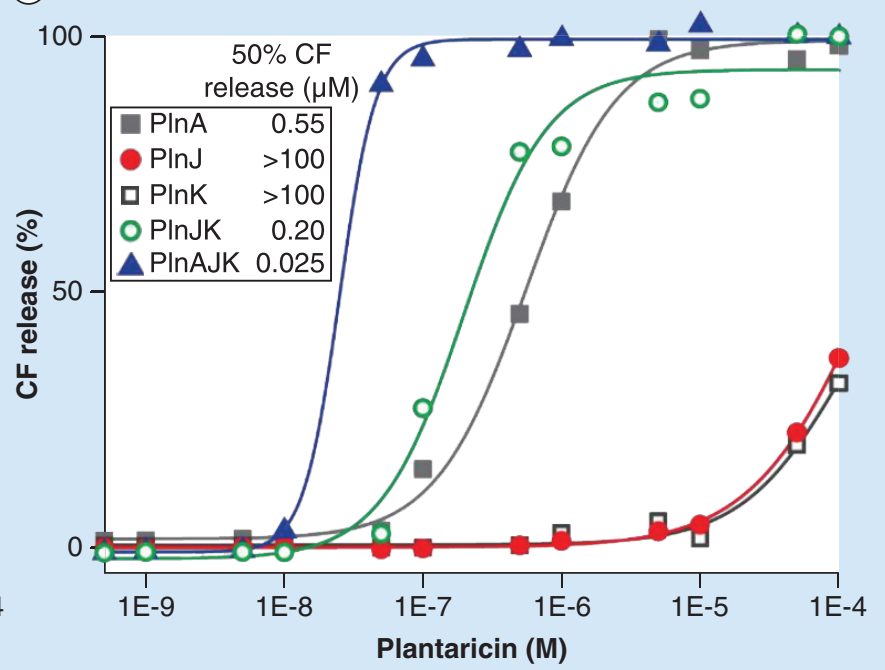

Figure 1. Antimicrobial activity of plantaricins. The antimicrobial activity of plantaricins was investigated against Staphylococcus epidermidis and verified using liposome model systems. The MIC and MBC values of (A) different combinations of PInA, E and F and (B) PInA, J and K against S. epidermidis ATCC 12228 was investigated after $20 \mathrm{~h}$ of incubation. In combination the respective peptides were added at the same concentration, for example, $50 \mu \mathrm{M}$ of PInEF was achieved by mixing $50 \mu \mathrm{M}$ of PlnE with $50 \mu \mathrm{M}$ PInF. The PInA alone showed antimicrobial activity, while PInE and F had antimicrobial effect only when combined together. Optimal activity of PInJ and $\mathrm{K}$ was observed when the two peptides were combined together. The CF release from liposomes was recorded 30 min after exposure to the same combinations of plantaricins to verify membrane permeabilization. The concentrations required to cause $50 \%$ CF release are indicated for (C) different combinations of PInA, E and F; and (D) PlnA, J and K. Three independent experiments were performed. CF: Carboxyfluorescein.

microscopy was utilized to visualize the nature of the damage of $S$. epidermidis ATCC following treatment with different Pln peptides at a final concentration of $25 \mu \mathrm{M}$ for $5 \mathrm{~min}$. Untreated bacteria showed typical morphology, where the outer cell wall (CW) and inner CM could be distinguished (Figure 3). Among the treatments with individual peptides alone, only $\mathrm{P} \ln \mathrm{A}$ and to some extent $\mathrm{PlnE}$ caused leakage of intracellular content. Interestingly, exposure of S. epidermidis to PlnEF or PlnJK, with and without PlnA, resulted in a significant alteration of bacterial ultrastructure and severe damage. The thickness of the $\mathrm{CW}$ was significantly increased and appeared loosen. Furthermore, the $\mathrm{CM}$ was completely detached and no longer tightly associated with the inner zone of the CW.

\section{Plantaricins act in synergy with antibiotics against $S$. epidermidis}

The plantaricin combinations EF and JK, respectively, were shown to be efficient against $S$. epidermidis ATCC by rapidly lysing the bacteria. Infections caused by Staphylococcus spp. are treated with different classes of antibiotics depending on their resistance patterns. Nonresistant bacteria are treated with, for example, the aminoglycoside antibiotics gentamicin or tetracycline that inhibits protein synthesis, while resistant bacteria are treated with gly- 

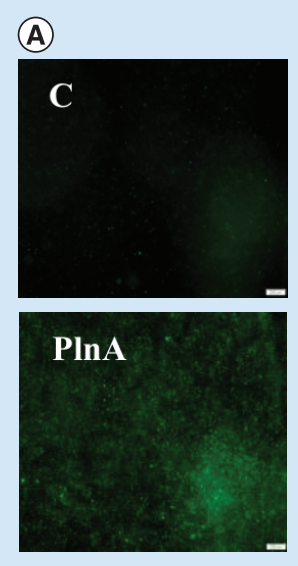

(B)

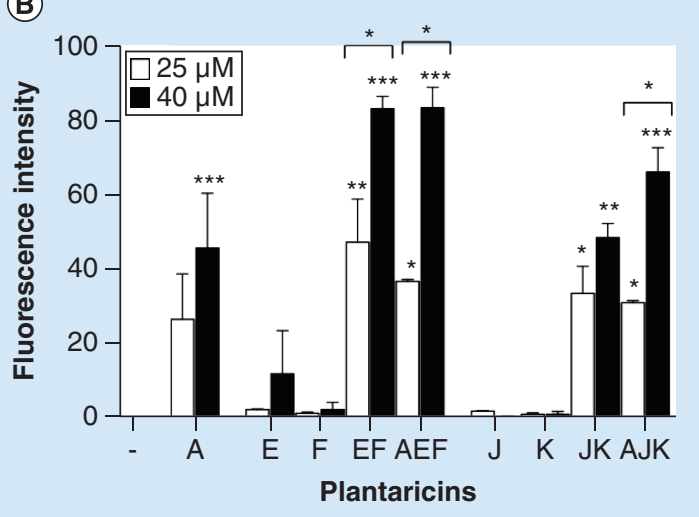

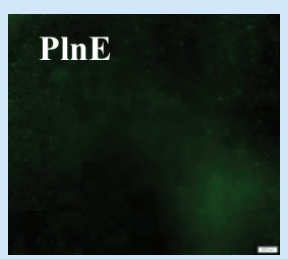
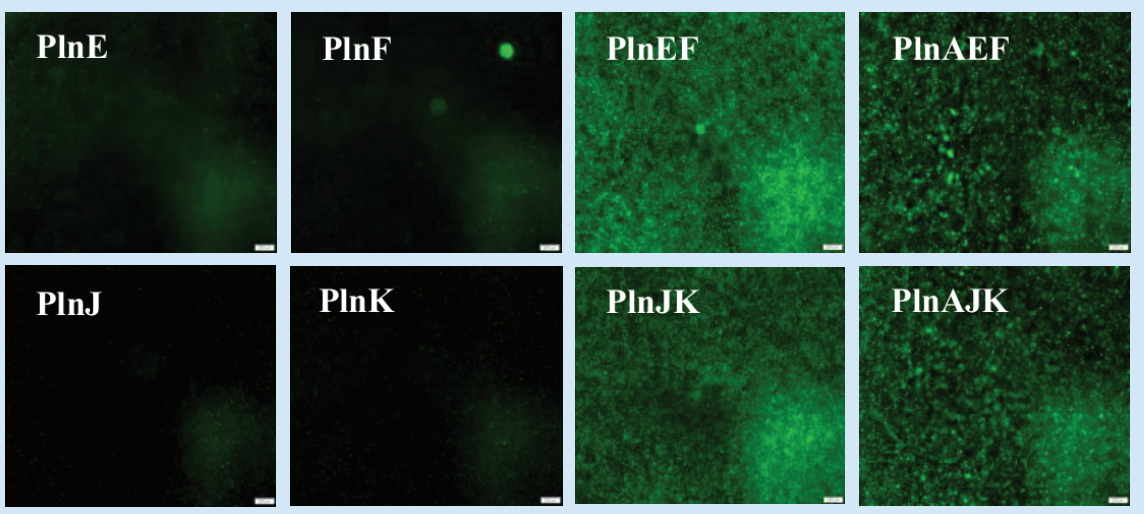

(c)

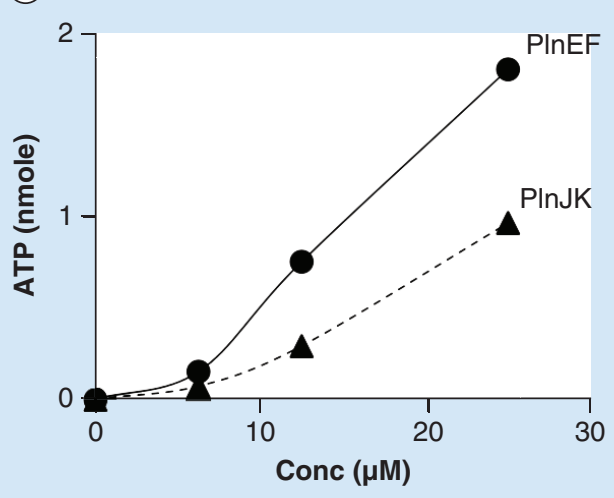

Figure 2. Rapid lysis of Staphylococcus epidermidis by plantaricins. Staphylococcus epidermidis was incubated with different combinations of plantaricins at a final concentration of 25 or $40 \mu \mathrm{M}$ for $2 \mathrm{~min}$. (A) Representative images of bacteria treated with $25 \mu \mathrm{M}$ of different Pln peptides are shown. Scale bar is $300 \mu \mathrm{m}$. (B) Fluorescence intensity of the images was quantified with the software ImageJ and results are presented as mean \pm SD. (C) Bacterial lysis was verified by quantifying ATP release from S. epidermidis ATCC following treatment with PInEF or PInJK. The bacteria were rapidly lysed (2-4 min) by PInEF and PInJK. The data are from three independent experiments.

PIn: Plantaricin; SD: Standard deviation.

copeptide antibiotics, including vancomycin and teicoplanin that interfere with CW synthesis. However, although glycopeptide antibiotics have been used restrictively, resistant bacteria have emerged through production of thicker CWs. This encouraged us to investigate potential synergistic inhibitory and bactericidal effects of plantaricins and antibiotics against S. epidermidis. A final and constant concentration of $12.5 \mu \mathrm{M}$ of PlnEF or PlnJK was tested together with antibiotics. Vancomycin and teicoplanin alone resulted in similar MIC $(3.1 \mu \mathrm{g} / \mathrm{ml})$ and MBC $(6.25 \mu \mathrm{g} / \mathrm{ml})$ values, while a concentration of $0.31 \mu \mathrm{g} / \mathrm{ml}$ of gentamicin was both inhibitory and bactericidal (Table 2A). The presence of PInEF reduced the MIC values of all three antibiotics by $>30$-fold, while PlnJK caused a reduction of twofold and approximately sevenfold of teicoplanin and gentamicin, respectively. The bactericidal activity of the combinatorial treatment, primarily with PlnEF, was significantly enhanced compared with antibiotics alone. Similar results were obtained with the laboratory reference strain S. epidermidis ATCC 12228 and S. epidermidis 126 isolated from an infected prosthetic joint. The latter strain is classified to have heterogeneous resistance to glycopeptide antibiotics is resistant to gentamicin (MIC $>5 \mu \mathrm{g} / \mathrm{ml}$ ). Addition of $12.5 \mu \mathrm{M}$ of PlnEF, but not PlnJK, significantly reduced the MIC and MBC values of gentamicin by 500 -fold and 260 -fold, respectively. Both S. epidermidis strains were found to be resistant to tetracycline (MIC $\geq 2 \mu \mathrm{g} / \mathrm{ml}$ ) and PlnEF significantly enhanced the inhibitory effect of tetracycline by 500 - and 125 -fold, for S. epidermidis ATCC and S. epidermidis 126, respectively. While PlnEF also lowered the bactericidal concentration of tetracycline, PlnJK was efficient at inhibiting the bacteria, but did not enhance the bactericidal activity. Furthermore, when the final concentration 

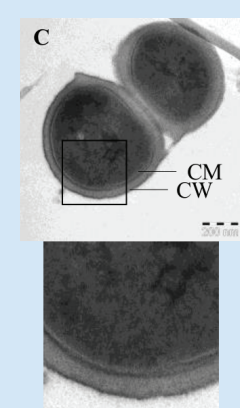

$56.2 \pm 7.2$

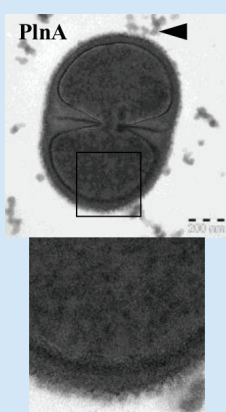

$66.4 \pm 7.1$

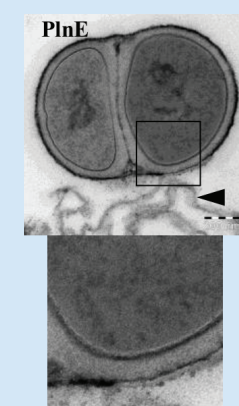

$67.2 \pm 11.9$

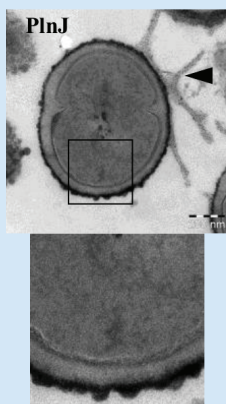

$64.8 \pm 8.8$

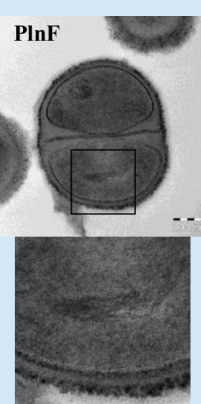

$58.3 \pm 6.2$

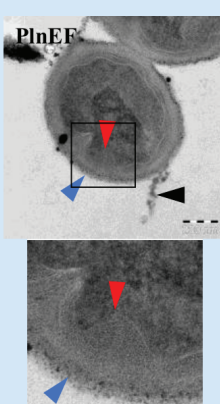

$98.3 \pm 11.8$

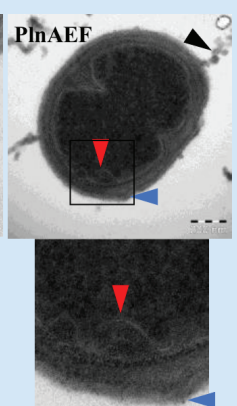

$96.7 \pm 9.1$

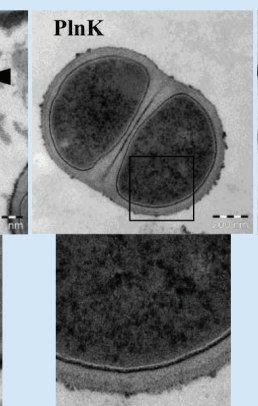

$63.3 \pm 5.3$

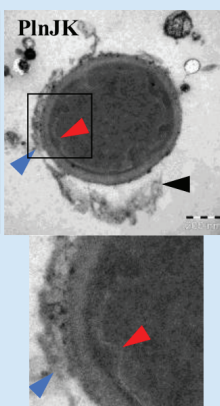

$89.6 \pm 9.9$

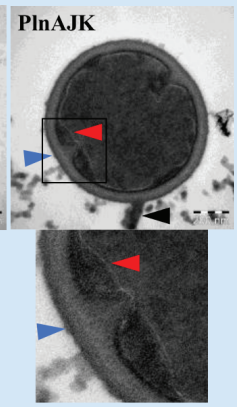

$92.9 \pm 9.6$

Figure 3. Exposure of Staphylococcus epidermidis to plantaricins causes severe ultrastructural changes.

Staphylococcus epidermidis was treated with $25 \mu \mathrm{M}$ of different plantaricins for $5 \mathrm{~min}$ and bacterial ultrastructure was examined using a Hitachi HT 7700 transmission electron microscope. The bacteria were severely affected, particularly by PInEF, AEF, JK and AJK. The inner CM was completely detached (red arrow heads) and leakage of intracellular content is observed (black arrow heads). The thickness of the CW was significantly increased (blue arrow heads), which was quantified and is represented by the values (mean \pm SD) under each panel. The lower image in each panel is a magnification of the selected area. Scale bar is $200 \mathrm{~nm}$.

CM: Cell membrane; CW: Cell wall; SD: Standard deviation.

of PlnEF was decreased to $6.25 \mu \mathrm{M}$, there was no synergistic effect with vancomycin, while the inhibitory and bactericidal effect with teicoplanin remained on both bacterial strains (Table 2B). A synergistic effect of PlnEF together with gentamicin was obtained only on S. epidermidis ATCC, while a synergistic effect of PlnEF with tetracycline was found against S. epidermidis 126.

\section{Discussion}

Staphylococcus epidermidis is an opportunistic pathogen and considered as one of the major causes of skin and soft-tissue infections in humans [23]. The resistance pattern of S. epidermidis includes several classes of antibiotics, such as methicillin, rifampicin, gentamicin and vancomycin [24], which causes limitations in treatment options. Similarly, treatment of MRSA infections with currently available antibiotics has been reported to be unsatisfactory, suggesting other antimicrobials and combination therapies as possible strategies [25]. Alternatives to antibiotics suggest bacteriocins as viable options [6,8]. Corr and colleagues [26] demonstrated that Lactobacillus salivarius efficiently protected mice from infections caused by Listeria monocytogenes. This inhibitory effect of pathogenic bacteria was directly associated with the ability of $L$. salivarius to produce the bacteriocin Abp118, as a mutation of this gene failed to protect the animals from L. monocytogenes infection. Using bacteriocins against skin and soft-tissue infections with less possibility to induce resistance may be advantageous, since bacterial resistance has increased the occurrence of complications in healthcare systems due to overuse of antibiotics [27]. This study shows that plantaricins of $L$. plantarum are effective against $S$. epidermidis when administered in conjunction with low concentrations of conventional antibiotics.

Plantaricins (A, E, F, J and K) are short peptides, ranging from 23 to 34 amino acids that possess antimicrobial characteristics. We show that both peptides of the same bacteriocin, in other words, PlnEF and PlnJK, are required 
Table 2. Plantaricins act synergistically with antibiotics.

\begin{tabular}{|c|c|c|c|c|}
\hline \multirow{3}{*}{ Antimicrobial agent } & \multicolumn{2}{|c|}{$\begin{array}{l}\text { S. epidermidis } \\
\text { ATCC } 12228\end{array}$} & \multicolumn{2}{|c|}{$\begin{array}{l}\text { S. epidermidis } \\
\text { hGISE }\end{array}$} \\
\hline & \multirow[t]{2}{*}{$\operatorname{MIC}(\mu \mathrm{g} / \mathrm{ml})$} & \multirow[t]{2}{*}{$\mathrm{MBC}(\mu \mathrm{g} / \mathrm{ml})$} & \multirow[t]{2}{*}{ MIC $(\mu \mathrm{g} / \mathrm{ml})$} & \multirow[t]{2}{*}{$\mathrm{MBC}(\mu \mathrm{g} / \mathrm{ml})$} \\
\hline & & & & \\
\hline Vancomycin/PInEF & $<0.097$ & 0.78 & $<0.097$ & $<0.097$ \\
\hline Vancomycin/PInJK & 3.1 & 3.1 & 3.1 & 3.1 \\
\hline Teicoplanin/PInJK & 1.5 & 3.1 & 1.5 & 6.25 \\
\hline Gentamicin & 0.31 & 0.31 & $>5$ & $>5$ \\
\hline Gentamicin/PInEF & $<0.0097$ & $<0.0097$ & $<0.0097$ & 0.019 \\
\hline Gentamicin/PInJK & 0.039 & 0.078 & 5 & $>5$ \\
\hline Tetracycline & $>8$ & $>8$ & 2 & 4 \\
\hline Vancomycin/PInEF & 3.1 & 3.1 & 3.1 & 3.1 \\
\hline Teicoplanin/PInEF & $<0.097$ & $<0.097$ & $<0.097$ & 0.39 \\
\hline Gentamicin/PInEF & 0.039 & 0.078 & $>5$ & $>5$ \\
\hline Tetracycline/PInEF & 4 & 4 & $<0.016$ & $<0.016$ \\
\hline \multicolumn{5}{|c|}{$\begin{array}{l}\text { (A) Staphylococcus epidermidis ATCC } 12228 \text { or } S \text {. epidermidis } 126 \text {, a clinical isolate that was found to have } \mathrm{hGISE} \text {, were exposed to a serial dilution of different an- } \\
\text { tibiotics alone or in combination with PInEF or PInJK at a final concentration of } 12.5 \mu \mathrm{M}(44 \mu \mathrm{g} / \mathrm{ml}) \text {. (B) MIC and MBC values of different antibiotics together with } \\
\text { PInEF at a final concentration of } 6.25 \mu \mathrm{M}(22 \mu \mathrm{g} / \mathrm{ml}) \text { toward S. epidermidis ATCC } 12228 \text { and S. epidermidis } 126 \text {. Three independent experiments were performed. } \\
\text { hGISE: Heterogeneous resistance to glycopeptide antibiotics; MBC: Minimal bactericidal concentration. }\end{array}$} \\
\hline
\end{tabular}

for efficient lysis of target bacterial membranes. These plantaricins belong to class IIb bacteriocins, and their activity has previously been reported to depend on two complementary peptides at equimolar concentrations [12]. Optimal antimicrobial activity of plantaricins is achieved by interaction of the two amphiphilic $\alpha$-helical peptides through GxxxG motifs and GxxxG-like motifs. This binding stabilizes the peptides and causes permeabilization of membranes, ultimately leading to death of susceptible bacteria due to alteration in intracellular $\mathrm{pH}$ and electric potential [13]. Interestingly, PlnA alone was efficient, both at lysing liposomes and inhibiting S. epidermidis growth. The function of $P \ln A$ was first described as a peptide pheromone that regulates transcription of genes within the $p \ln$ locus, including $p \ln E F I$ and $p \ln J K L R$; however, it is now established that this peptide also displays antimicrobial activity [28,29]. Furthermore, we show that the two-peptide bacteriocins PlnEF and PlnJK are remarkably rapid at permeabilizing $S$. epidermidis. Total 2 min of exposure was sufficient to allow Sytox Green to accumulate and significantly fluoresce through DNA-binding, and ATP release peaked at 1-4 min post-treatment. Analysis of bacterial ultrastructure revealed an increased thickness of the $\mathrm{CW}$ that appeared loose, while the inner $\mathrm{CM}$ was completely detached and distorted. Thickening of the CW has been documented for several conventional antibiotics, such as $\beta$-lactam antibiotics, vancomycin, rifampicin and tetracycline (reviewed in [30]). These effects are observed first after $\geq 2 \mathrm{~h}$ of treatment with low concentrations of antibiotic. The $\mathrm{CW}$ enlargement by antibiotics targeting protein synthesis has been suggested to be due to inhibition of peptidoglycan hydrolases that are needed to loosen the $\mathrm{CW}$, which in addition to continued production and incorporation of peptidoglycan precursor, ultimately results in thickening of the CW [30]. Two-peptide bacteriocins, including PlnEF and PlnJK, target bacterial membranes to be permeable to small molecules [31]. While PlnEF creates pores followed by leakage of monovalent cations, PlnJK causes leakage of anions [32]. This may lead to a perturbed electrolyte balance with accompanying efflux of water that affects the tight association of CM with the inner wall zone, and eventually the thickness of the CW. However, more research is needed to understand the precise mechanisms underlying these dramatic effects. Both PlnEF and PlnJK have been reported to form pores on target membranes, but their mechanism of action and affinity is different toward different microorganisms [13,28].

Staphylococcus spp. are responsible for causing skin and soft tissue infections that may be life threatening. Although vancomycin is an effective treatment, heteroresistance to this antibiotic is increasing [33] and biofilm formation 
of staphylococci has been reported to decrease the activity of vancomycin [34]. It would be advantageous to use agents that efficiently permeabilized bacterial CWs in combination with low doses of conventional antibiotics to improve the bactericidal effects. The permeabilizing and antimicrobial activity of plantaricins together with different antibiotics was investigated. The PlnEF dramatically enhanced the effects of vancomycin and teicoplanin by $>30$-fold, and gentamicin and tetracycline by $>500$-fold, while PlnJK was less effective. Plantaricin-induced disruption of the CW and CM may facilitate efficient penetration and accumulation of antibiotics, thus rapidly reaching a critical concentration with inhibitory and bactericidal effect. The concept of combination therapy has been implicated to mainly increase the likelihood of providing an effective agent rather than contributing to synergistic effects between two or more agents [14,35]. In vitro data may therefore be useful to support the choice of appropriate treatments when providing combination therapy [36]. Gimeno and colleagues showed that the combination of cefotaxime and ofloxacin acted synergistically against 55.8\% of the isolated S. aureus strains [37]. However, although the mechanism of antimicrobial activity of the combination antibiotic/bacteriocin differs from antibiotic/antibiotic, more research is needed to support potential applications in clinical settings.

\section{Conclusion}

In this study, we show that PlnEF act in synergy with gentamicin, tetracycline, vancomycin and teicoplanin against S. epidermidis. Sub-MIC concentrations of PlnEF were most effective in significantly reducing the concentrations of gentamicin, tetracycline and teicoplanin. This study suggests that combination therapies composed of plantaricins and conventional antibiotics should be considered and further developed as potential treatment against $S$. epidermidis. Although these results are encouraging, additional antibiotics and bacterial strains have to be tested, and the cytotoxicity and stability of plantaricins have to be fully characterized.

\section{Summary points}

- Plantaricin EF and JK of Lactobacillus plantarum are efficient at permeabilizing Staphylococcus epidermidis.

- The permeabilizing activity of plantaricins was verified using liposome model systems.

- The mechanism PInEF and PInJK involves disruption of the bacterial cell membrane.

- Bacteria are rapidly lysed (2 $\mathrm{min}$ ) as indicated by the instant release of ATP.

- The pheromone peptide PInA enhances the antimicrobial effect of PInEF and PInJK.

- Plantaricins act synergistically with traditional antibiotics, enhancing their effects by 30 - to 500 -fold.

\section{Supplementary data}

To view the supplementary data that accompany this paper please visit the journal website at: www.futuremedicine.com/doi/full/10.2217/fmb-2018-0285

\section{Author contributions}

Design of the work was performed by $\mathrm{T}$ Bengtsson and $\mathrm{H}$ Khalaf. Acquisition of data, analysis and interpretation, drafting and critical revision, approval of the final version and agreement to be accountable for the work were performed by $\mathrm{R}$ Selegård, $\mathrm{A}$ Musa, P Nyström, D Aili, T Bengtsson and H Khalaf.

Financial \& competing interests disclosure

This work was supported by the Foundation of Magnus Bergvall (grant number 2015-00823), the Knowledge Foundation (grant numbers 20150244, 20150086), and Sweden and Swedish Research Council (grant number 2016-04874). The authors have no other relevant affiliations or financial involvement with any organization or entity with a financial interest in or financial conflict with the subject matter or materials discussed in the manuscript apart from those disclosed.

No writing assistance was utilized in the production of this manuscript.

\section{Open access}

This work is licensed under the Attribution-NonCommercial-NoDerivatives 4.0 Unported License. To view a copy of this license, visit http://creativecommons.org/licenses/by-nc-nd/4.0/ 


\section{References}

Papers of special note have been highlighted as: $\bullet$ of interest; $\bullet \bullet$ of considerable interest

1. Joerger RD. Alternatives to antibiotics: bacteriocins, antimicrobial peptides and bacteriophages. Poult. Sci. 82(4), 640-647 (2003).

2. Assis LM, Nedeljkovic M, Dessen A. New strategies for targeting and treatment of multi-drug resistant Staphylococcus aureus. Drug Resist. Updates 31, 1-14 (2017).

3. Fridkin SK, Hageman J, Mcdougal LK et al. Epidemiological and microbiological characterization of infections caused by Staphylococcus aureus with reduced susceptibility to vancomycin, United States, 1997-2001. Clin. Infect. Dis. 36(4), 429-439 (2003).

4. Kali A. Antibiotics and bioactive natural products in treatment of methicillin resistant Staphylococcus aureus: a brief review. Pharmacogn. Rev. 9(17), 29-34 (2015).

5. Grigg C, Palms D, Stone ND et al. Burden of invasive methicillin-resistant staphylococcus aureus infections in nursing home residents. J. Am. Geriatr. Soc. doi:10.1111/jgs.15451 (2018) (Epub ahead of print).

6. Cotter PD, Ross RP, Hill C. Bacteriocins - a viable alternative to antibiotics? Nat. Rev. Microbiol. 11(2), 95-105 (2013).

-• Highlights the importance of bacteriocins as the next generation of antimicrobial substances against infections.

7. Czaplewski L, Bax R, Clokie M et al. Alternatives to antibiotics-a pipeline portfolio review. Lancet Infect. Dis. 16(2), 239-251 (2016).

-. An overview of alternative strategies for treatment of antibiotic resistant bacterial infections.

8. Diep DB, Straume D, Kjos M, Torres C, Nes IF. An overview of the mosaic bacteriocin pln loci from Lactobacillus plantarum. Peptides 30(8), 1562-1574 (2009).

- Describes the organization of pln genes within the pln locus of the Lactobacillus plantarum genome.

9. Yang SC, Lin CH, Sung CT, Fang JY. Antibacterial activities of bacteriocins: application in foods and pharmaceuticals. Front. Microbiol. 5, 241 (2014).

10. Khalaf H, Nakka SS, Sanden C et al. Antibacterial effects of Lactobacillus and bacteriocin PLNC8 alphabeta on the periodontal pathogen Porphyromonas gingivalis. BMC Microbiol. 16(1), 188 (2016).

- Shows for the first time that $L$. plantarum and its bacteriocin PLNC8 $\alpha \beta$ efficiently inhibits the periodontal pathogen Porphyromonas gingivalis.

11. Bengtsson T, Zhang B, Selegard R, Wiman E, Aili D, Khalaf H. Dual action of bacteriocin PLNC8 alphabeta through inhibition of Porphyromonas gingivalis infection and promotion of cell proliferation. Pathog. Dis. 75(5), ftx064, 1-10 (2017).

- $\quad$ PLNC8 $\alpha \beta$ antagonizes the cytotoxic effect of $P$. gingivalis and stimulates proliferation of gingival fibroblasts.

12. Ekblad B, Kyriakou PK, Oppegard C, Nissen-Meyer J, Kaznessis YN, Kristiansen PE. Structure-function analysis of the two-peptide bacteriocin plantaricin EF. Biochemistry 55(36), 5106-5116 (2016).

- The insertion, structure and orientation of plantaricin EF is shown using molecular dynamics simulations.

13. Oppegard C, Kjos M, Veening JW, Nissen-Meyer J, Kristensen T. A putative amino acid transporter determines sensitivity to the two-peptide bacteriocin plantaricin JK. Microbiol. Open 5(4), 700-708 (2016).

- Suggests that APC superfamily transporter is a possible target for plantaricin JK.

14. Tamma PD, Cosgrove SE, Maragakis LL. Combination therapy for treatment of infections with Gram-negative bacteria. Clin. Microbiol. Rev. 25(3), 450-470 (2012).

15. Worthington RJ, Melander C. Combination approaches to combat multidrug-resistant bacteria. Trends Biotechnol. 31(3), 177-184 (2013).

16. Chi H, Holo H. Synergistic antimicrobial activity between the broad spectrum bacteriocin garvicin KS and Nisin, Farnesol and Polymyxin B against gram-positive and gram-negative bacteria. Curr. Microbiol. doi:10.1007/s00284-017-1375-y (2017) (Epub ahead of print).

17. Zhao X, Zhen Z, Wang X, Guo N. Synergy of a combination of nisin and citric acid against Staphylococcus aureus and Listeria monocytogenes. Food Addit. Contam. Part A Chem. Anal. Control Expo. Risk Assess. 34(12), 2058-2068 (2017).

18. Field D, R OC, Cotter PD, Ross RP, Hill C. In vitro activities of Nisin and Nisin derivatives alone and in combination with antibiotics against staphylococcus biofilms. Front. Microbiol. 7, 508 (2016).

19. Mollica M, Hyman Z. Professional development utilizing an oncology summer nursing internship. Nurse Educ. Pract. 16(1), 188-192 (2016).

20. Momen S, Mallipeddi R, Al-Niaimi F. The use of lasers in Becker's naevus: an evidence-based review. J. Cosmet. Laser Ther. 18(4), 188-192 (2016).

21. Wang $\mathrm{Q}$, Chen $\mathrm{Z}, \mathrm{Xu} \mathrm{Y}$. [The relationship between onset of labour and concentration of hyaluronic acid and estradiol to progesterone ratio]. Zhonghua Fu Chan Ke Za Zhi 31(8), 477-479 (1996).

22. Hauge HH, Mantzilas D, Eijsink VG, Nissen-Meyer J. Membrane-mimicking entities induce structuring of the two-peptide bacteriocins plantaricin E/F and plantaricin J/K. J. Bacteriol. 181(3), 740-747 (1999).

23. Rogers KL, Fey PD, Rupp ME. Coagulase-negative staphylococcal infections. Infect. Dis. Clin. N. Am. 23(1), 73-98 (2009). 
24. Otto M. Staphylococcus epidermidis: the 'accidental' pathogen. Nat. Rev. Microbiol. 7(8), 555-567 (2009).

25. Nguyen HM, Graber CJ. Limitations of antibiotic options for invasive infections caused by methicillin-resistant Staphylococcus aureus: is combination therapy the answer? J. Antimicrob. Chemother. 65(1), 24-36 (2010).

26. Corr SC, Li Y, Riedel CU, O'toole PW, Hill C, Gahan CG. Bacteriocin production as a mechanism for the antiinfective activity of Lactobacillus salivarius UCC118. Proc. Natl Acad. Sci. USA 104(18), 7617-7621 (2007).

- Demonstrates that $L$. salivarius efficiently protects mice from infections caused by Listeria monocytogenes, which is directly associated with the ability of $L$. salivarius to produce the bacteriocin Abp118.

27. Blaser M. Antibiotic overuse: stop the killing of beneficial bacteria. Nature 476(7361), 393-394 (2011).

28. Anderssen EL, Diep DB, Nes IF, Eijsink VG, Nissen-Meyer J. Antagonistic activity of Lactobacillus plantarum C11: two new two-peptide bacteriocins, plantaricins EF and JK, and the induction factor plantaricin A. Appl. Environ. Microbiol. 64(6), 2269-2272 (1998).

29. Kristiansen PE, Fimland G, Mantzilas D, Nissen-Meyer J. Structure and mode of action of the membrane-permeabilizing antimicrobial peptide pheromone plantaricin A. J. Biol. Chem. 280(24), 22945-22950 (2005).

30. Cushnie TP, O'driscoll NH, Lamb AJ. Morphological and ultrastructural changes in bacterial cells as an indicator of antibacterial mechanism of action. Cell. Mol. Life Sci. 73(23), 4471-4492 (2016).

31. Sharma A, Srivastava S. Anti-Candida activity of two-peptide bacteriocins, plantaricins (Pln $\mathrm{E} / \mathrm{F}$ and $\mathrm{J} / \mathrm{K}$ ) and their mode of action. Fungal Biol. 118(2), 264-275 (2014).

32. Moll GN, Van Den Akker E, Hauge HH et al. Complementary and overlapping selectivity of the two-peptide bacteriocins plantaricin EF and JK. J. Bacteriol. 181(16), 4848-4852 (1999).

33. Mashaly GE, El-Mahdy RH. Vancomycin heteroresistance in coagulase negative Staphylococcus blood stream infections from patients of intensive care units in Mansoura University Hospitals, Egypt. Ann. Clin. Microbiol. Antimicrob. 16(1), 63 (2017).

34. Zhang Y, Fu Y, Yu J et al. Synergy of ambroxol with vancomycin in elimination of catheter-related Staphylococcus epidermidis biofilm in vitro and in vivo. J. Infect. Chemother. 21(11), 808-815 (2015).

35. Garnacho-Montero J, Sa-Borges M, Sole-Violan J et al. Optimal management therapy for Pseudomonas aeruginosa ventilator-associated pneumonia: an observational, multicenter study comparing monotherapy with combination antibiotic therapy. Crit. Care Med. 35(8), 1888-1895 (2007).

36. Tangden T. Combination antibiotic therapy for multidrug-resistant Gram-negative bacteria. Upsala J. Med. Sci. 119(2), 149-153 (2014).

37. Gimeno C, Borja J, Navarro D, Valdes L, Garcia-Barbal J, Garcia-De-Lomas J. In vitro interaction between ofloxacin and cefotaxime against Gram-positive and gram-negative bacteria involved in serious infections. Chemotherapy 44(2), 94-98 (1998). 
